\title{
Biomasse et stocks de carbone des forêts tropicales africaines (synthèse bibliographique)
}

\author{
Grace Jopaul Loubota Panzou ${ }^{(1,2)}$, Jean-Louis Doucet ${ }^{(1)}$, Jean-Joël Loumeto ${ }^{(2)}$, \\ Achille Biwole ${ }^{(1,3)}$, Sébastien Bauwens ${ }^{(1)}$, Adeline Fayolle ${ }^{(1)}$ \\ (1) Université de Liège - Gembloux Agro-Bio Tech. Département BIOSE. Axe de Gestion des Ressources forestières - \\ Foresterie tropicale. Passage des Déportés, 2. BE-5030 Gembloux (Belgique). E-mail : loubotagrace@gmail.com \\ (2) Université Marien Ngouabi. Faculté des Sciences et Techniques. Laboratoire de Botanique et Écologie. B.P. 69. \\ Brazzaville (République du Congo). \\ (3) Université de Douala. École Normale Supérieure d'Enseignement Technique. B.P. 1872. Douala (Cameroun).
}

Reçu le 14 octobre 2015, accepté le 16 juin 2016.

Introduction. Quantifier les stocks de biomasse et de carbone contenus dans les forêts tropicales est devenu une priorité internationale dans le cadre de la mise en œuvre du mécanisme REDD+. La biomasse forestière est estimée à trois échelles spatiales successives : l'arbre, le peuplement et la région. Cet article propose une synthèse des connaissances sur l'estimation de la biomasse et des stocks de carbone contenus dans les forêts tropicales africaines.

Littérature. Cette synthèse bibliographique démontre que peu d'équations allométriques, équations qui permettent d'estimer la biomasse de l'arbre à partir de mesures non destructives (diamètre, hauteur), ont été établies pour les forêts tropicales africaines. Au niveau du peuplement, cette synthèse met en exergue les variations spatiales et temporelles connues de la biomasse entre les types de forêts d'Afrique tropicale. Si la reconstitution de la biomasse après une perturbation (l'exploitation forestière, par exemple) est relativement rapide, il existe encore beaucoup d'incertitudes sur les variations spatiales de la biomasse et il n'y a pas de consensus sur une cartographie régionale de la biomasse. La qualité de la cartographie de la biomasse dépend fortement des différents capteurs utilisés (optique, RADAR ou LiDAR) et de l'équation allométrique utilisée pour convertir les données d'inventaires forestiers en biomasse.

Conclusions. Considérant le manque de précision des équations allométriques disponibles et des données d'inventaires forestiers à large échelle spatiale, il y a encore beaucoup d'incertitudes sur les estimations de la biomasse et des stocks de carbone contenus dans les forêts tropicales africaines.

Mots-clés. Biomasse, carbone, forêt tropicale, allométrie, inventaire forestier, télédétection, Afrique tropicale.

\section{Biomass and carbon stocks of tropical African forests. A review}

Introduction. Quantifying the biomass and carbon stocks contained in tropical forests has become an international priority for the implementation of the REDD+ mechanism. Forest biomass is estimated at three successive levels: the tree, the stand and the region level. This paper reviews the state of the art regarding the estimation of biomass and carbon stocks in tropical African forests.

Literature. This review highlights the fact that very few allometric equations, equations used for estimating the biomass of the tree using non-destructive measurements (diameter, height), have been established for tropical African forests. At the stand level, the review highlights the spatial and temporal variations in biomass between forest types in Central and Eastern Africa. While biomass recovery after a disturbance (logging, for instance) is rather quick, a great deal of uncertainty still remains regarding the spatial variation in biomass, and there is no consensus on a regional biomass map. The quality of biomass mapping in tropical Africa strongly depends on the type of remotely-sensed data being used (optical, RADAR or LIDAR), and the allometric equation used to convert forest inventory data into biomass.

Conclusions. Based on the lack of precision of the available allometric equations and forest inventory data and the large spatial scale involved, many uncertainties persist in relation to the estimation of the biomass and carbon stocks contained in African tropical forests.

Keywords. Biomass, carbon, tropical forests, allometry, forest inventories, remote sensing, tropical Africa. 


\section{INTRODUCTION}

Les forêts tropicales contiennent 40 à $50 \%$ du carbone terrestre et jouent un rôle majeur dans le cycle global du carbone (Pan et al., 2011). La perte du couvert forestier résultant de la déforestation et de la dégradation de ces forêts contribue à environ 10-15\% des émissions mondiales annuelles de gaz à effet de serre (van der Werf et al., 2009). La Convention-Cadre des Nations Unies sur les Changements Climatiques a examiné la possibilité de réduire ces émissions à travers l'initiative internationale REDD+ (Réduction des Émissions dues à la Déforestation et la Dégradation des forêts). Le mécanisme REDD+ vise à encourager les pays en développement à préserver les massifs forestiers moyennant des compensations financières issues des crédits de carbone (Angelsen et al., 2013). Toutefois, sa mise en œuvre dépend d'un système de Mesure, Notification et Vérification (MNV ou MRV en anglais) concernant l'estimation de la biomasse et des stocks de carbone contenus dans les forêts (Gibbs et al., 2007 ; Saatchi et al., 2011 ; Baccini et al., 2012 ; Clark et al., 2012).

Dans les écosystèmes forestiers, le carbone est stocké dans la biomasse vivante (biomasse aérienne et biomasse souterraine), le bois mort, la matière organique du sol et la litière (GIEC, 2003). Renfermant plus de 3/4 du carbone de la forêt, la biomasse vivante, objet spécifique de cette synthèse, est estimée via trois échelles spatiales successives (Chave et al., 2004 ; Clark et al., 2012 ; Molto et al., 2013). Au niveau de l'arbre, les données de diamètre et éventuellement de hauteur issues d'inventaires forestiers sont converties en biomasse par le biais d'une équation allométrique. Au niveau du peuplement, les biomasses individuelles sont sommées par unité de surface inventoriée (parcelle forestière) et une biomasse moyenne est calculée par type de peuplement. Grâce aux estimations de biomasse des parcelles forestières, il est ensuite possible de spatialiser les estimations de biomasse à l'aide de données de télédétection (Goetz et al., 2009 ; Bastin et al., 2014). Cette spatialisation peut être opérée selon différentes approches et à différentes échelles pour produire in fine des cartes de biomasse.

Il existe, cependant, des sources d'erreur à chacune de ces étapes (Chave et al., 2004 ; Gibbs et al., 2007). À titre d'exemple, le manque à la fois de données d'inventaire forestier à large échelle spatiale et de modèles allométriques appropriés pour convertir ces données d'inventaire en biomasse est souvent cité comme un handicap majeur dans la précision des estimations de biomasse et de stocks de carbone. Le présent article dresse l'état actuel des connaissances sur l'estimation de la biomasse et des stocks de carbone contenus dans les forêts tropicales africaines. Les méthodes d'estimation de la biomasse à l'échelle de l'arbre sont d'abord présentées. Ensuite, les variations spatiales et temporelles de la biomasse à l'échelle du peuplement sont abordées. Enfin, les cartes de biomasse actuellement disponibles sont discutées. Ces éléments permettent d'identifier les lacunes majeures en Afrique tropicale et les priorités de recherches futures pouvant faciliter une mise en œuvre effective du mécanisme REDD+.

La recherche documentaire a été opérée en utilisant les bases de données Scopus, Google scholar, Collection ULg et BASE (Bielefeld Academic Search Engine). Les mots clés suivants en français - et leur équivalent en anglais - ont été utilisés : biomasse aérienne, biomasse souterraine, forêt tropicale, Afrique, modèles/équations allométriques, stock de carbone, carte de biomasse.

\section{ESTIMATION DE LA BIOMASSE AU NIVEAU DE L'ARBRE}

\subsection{L'allométrie}

L'allométrie se définit comme la relation entre deux (ou plus) caractéristiques de taille d'un arbre (Picard et al., 2012). Actuellement, il existe deux approches pour estimer la biomasse d'un arbre en Afrique tropicale. Toutes les deux reposent sur l'utilisation des relations allométriques. En foresterie, les relations allométriques concernent le diamètre, la hauteur, les dimensions du houppier, le volume et la biomasse de l'arbre (King, 1996).

La première approche est basée sur des équations allométriques qui permettent d'estimer directement la biomasse totale ou partielle (biomasse aérienne, biomasse souterraine, etc.) d'un arbre en fonction de prédicteurs. Les prédicteurs les plus importants de la biomasse sont, par ordre décroissant : le diamètre du tronc, la densité du bois et la hauteur totale (Chave et al., 2005). Deux études récentes menées dans le bassin amazonien (Goodman et al., 2014) et dans le bassin du Congo (Ploton et al., 2016) indiquent que la prise en compte des dimensions du houppier améliore significativement les estimations de biomasse.

La seconde approche est une méthode indirecte qui utilise un tarif de cubage pour convertir le diamètre, et éventuellement la hauteur du tronc, en volume du tronc puis prend en compte la densité du bois pour convertir le volume du tronc en biomasse du tronc. Cette dernière est ensuite étendue à la biomasse aérienne totale via un facteur d'expansion de la biomasse (Brown et al., 1989 ; Maliro et al., 2010).

L'estimation de la biomasse d'un arbre s'accompagne toujours d'une erreur qui correspond à l'écart entre les valeurs de biomasse observées et les valeurs prédites par le modèle allométrique. L'erreur 
sur la biomasse de l'arbre peut être décomposée en trois catégories (Chave et al., 2004 ; van Breugel et al., 2011 ; Molto et al., 2013) :

- l'erreur due au choix de l'équation allométrique ;

- l'erreur de prédiction du modèle qui comprend l'incertitude sur les coefficients du modèle et l'erreur résiduelle du modèle ;

- l'erreur sur les prédicteurs qui comprend les erreurs de mesure des variables dendrométriques (diamètre, hauteur, etc.) et celles relatives à la densité du bois, liées à la détermination de l'espèce, aux mesures en laboratoire ou à la variabilité intra-spécifique.

Parmi ces types d'erreurs, le choix de l'équation allométrique est la source d'erreur la plus importante dans les estimations de la biomasse (Chave et al., 2004; Molto et al., 2013).

En Afrique tropicale, il a été montré que le choix de l'équation allométrique contribue à environ $76 \%$ de l'erreur totale dans les estimations de la biomasse de l'arbre (Moundounga Mavouroulou et al., 2014 ; Picard et al., 2015). Cette erreur élevée provient des équations allométriques pantropicales (modèles calibrés sur les données de biomasse de toutes les régions et de tous les types de forêts tropicales) et des équations allométriques locales (modèles calibrés sur les données de biomasse d'un type/site spécifique de forêt tropicale).

\subsection{Les équations allométriques pantropicales}

Jusqu'à récemment, en l'absence d'équations allométriques calibrées localement, des équations pantropicales étaient utilisées en Afrique tropicale. Dans une première approche, Brown et al. (1989) puis Chave et al. (2005) ont développé des équations allométriques pantropicales séparément pour les forêts de type «Dry » (pluviométrie < $1500 \mathrm{~mm}$, saison sèche $>5$ mois), «Moist» (pluviométrie 1500-3500 mm, saison sèche 1-5 mois) et «Wet» (pluviométrie $>3500 \mathrm{~mm}$, saison sèche $<1$ mois).

Les équations pantropicales développées par Chave et al. (2005) ont largement été utilisées en Afrique tropicale. Ces équations ont permis de convertir les données de diamètre, issues d'inventaires forestiers, et de densité du bois, provenant de bases de données, en biomasse aérienne à l'échelle des arbres (Stephenson et al., 2014) et des forêts (Djuikouo et al., 2010 ; Gourlet-Fleury et al., 2011; Makana et al., 2011 ; Maniatis et al., 2011 ; Medjibe et al., 2011 ; GourletFleury et al., 2013 ; Bastin et al., 2015 ; Ekoungoulou et al., 2015). Dans certains travaux, la hauteur totale de l'arbre était également incorporée dans le jeu de prédicteurs. La hauteur était alors soit mesurée (Djomo et al., 2011 ; Shirima et al., 2011 ; Marshall et al., 2012 ; Day et al., 2013 ; Lindsell et al., 2013 ; Bastin et al.,
2014 ; Bastin et al., 2015 ; Ensslin et al., 2015 ; Gatti et al., 2015 ; Shirima et al., 2015), soit estimée à partir d'une relation allométrique hauteur - diamètre établie à l'échelle mondiale (Lewis et al., 2009), régionale (Lewis et al., 2013) ou locale (Kearsley et al., 2013 ; Bastin et al., 2015 ; Shirima et al., 2015 ; Fayolle et al., 2016).

Toutefois, la validité des équations pantropicales de Chave et al. (2005) en Afrique tropicale a été fortement discutée. La limite majeure serait liée à l'absence de données provenant d'Afrique tropicale dans la calibration des équations. En ce qui concerne les forêts de type «Dry», les prédictions, testées dans trois sites du nord-est de la Tanzanie dans les forêts claires de type Miombo sur 167 arbres répartis entre 1 et $110 \mathrm{~cm}$ de diamètre, montrent une sous-estimation systématique de la biomasse des arbres de l'ordre de 10 à $20 \%$ (Mugasha et al.,2013). Dans les forêts de type « Moist », l'équation a été validée au sud-est du Cameroun dans une forêt dense humide de transition entre les types de forêt sempervirent et semi-caducifolié sur 138 arbres répartis entre 5 et $192 \mathrm{~cm}$ de diamètre (Fayolle et al., 2013). Le modèle a aussi été validé au nord-est de la République Démocratique du Congo (RDC) dans les forêts denses humides semi-caducifoliées sur 12 arbres répartis entre 24 et $52 \mathrm{~cm}$ de diamètre (Ebuy et al., 2011). Par contre, des biais importants de 10 et $40 \%$ ont été identifiés respectivement dans les forêts sempervirentes du Ghana sur 42 arbres répartis entre 2 à $180 \mathrm{~cm}$ de diamètre (Henry et al., 2010) et dans les forêts de transition du nord-est du Gabon sur 101 arbres répartis entre 11 à $109 \mathrm{~cm}$ de diamètre (Ngomanda et al., 2014).

Dans une seconde approche, Feldpausch et al. (2012) ont proposé deux équations pantropicales de type «Moist» incorporant des données de biomasse pour 116 arbres d'Afrique tropicale dans leur construction. Une équation allométrique pantropicale à deux prédicteurs, le diamètre et la densité du bois (équation 1), et une autre équation à trois prédicteurs, le diamètre, la densité du bois et la hauteur totale (équation 2), ont été établies. Enfin, Chave et al. (2014) ont récemment effectué une révision des équations allométriques pantropicales en intégrant les données de biomasse pour 4004 arbres dont 1006 arbres d'Afrique tropicale. Une équation allométrique pantropicale à trois prédicteurs (diamètre, hauteur totale et densité $\mathrm{du}$ bois), valable pour tous les types de forêts tropicales (équation 3 ) et une équation dépendant du diamètre, de la densité du bois et d'un facteur de stress environnemental $\mathrm{E}$ (équation 4) ont ainsi pu être établies. En Afrique tropicale, les équations de Feldpausch et al. (2012) ont été utilisées dans les travaux de Slik et al. (2013) et celles de Chave et al. (2014) dans les travaux de Doetterl et al. (2015) en RDC, de Lung et al. (2015) au Kenya et de Fayolle 
et al. (2016) au Cameroun. Les équations allométriques développées par Chave et al. (2014) (équations 3 et 4) sont plus performantes et robustes que les équations allométriques développées par Feldpausch et al. (2012) (équations 1 et 2) et l'utilisation de ces équations (3 et 4) conduit à une précision de $90 \%$ dans les estimations de la biomasse à l'échelle de 0,25 ha dans les forêts tropicales humides (Chave et al., 2014). les équations sont construites à partir d'un nombre limité d'espèces (Kuyah et al., 2012a ; Kuyah et al., 2012b ; Chidumayo, 2013).

Seulement trois études menées en Afrique tropicale suivent les recommandations de Chave et al. (2004). Deux études réalisées en Afrique centrale ont permis d'établir des équations locales pour l'estimation de la biomasse aérienne des arbres des forêts denses humides de transition du sudest du Cameroun $\mathrm{AGB}=\exp \left(-1,822+2,337 \times \ln \mathrm{D}+0,163 \times(\operatorname{lnD})^{2}-0,024 \times(\operatorname{lnD})^{3}+0,979 \times \ln \varrho\right)$ $\mathrm{AGB}=\exp \left(-2,920+0,989 \times \ln \left(\mathrm{\varrho D}^{2} \mathrm{H}\right)\right)$

$\mathrm{AGB}=0,0673 \times\left(\mathrm{\varrho D}^{2} \mathrm{H}\right)^{0,976}$

$\left.\mathrm{AGB}=\exp \left(-1,803-0,976 \times \mathrm{E}+0,976 \times \ln \varrho+2,673 \times \ln \mathrm{D}-0,0299 \times(\ln \mathrm{D})^{2}\right)\right)$

(Fayolle et al., 2013) et du nord-est du Gabon (Ngomanda et al., 2014). Une études a été réalisée en Afrique de l'Est

où $\mathrm{AGB}, \varrho, \mathrm{D}, \mathrm{H}$ et $\mathrm{E}$ sont respectivement la biomasse aérienne (en $\mathrm{kg}$ ), la densité du bois spécifique (en $\mathrm{g} \cdot \mathrm{cm}^{-3}$ ), le diamètre de l'arbre $(\mathrm{en} \mathrm{cm})$, la hauteur totale de l'arbre (en $\mathrm{m}$ ) et une variable qui quantifie le stress environnemental développé par Chave et al. (2014). Le facteur de stress environnemental E englobe la saisonnalité de la température, l'intensité de la sècheresse et la saisonnalité des précipitations et varie entre $-0,2$ et 1 à travers les forêts tropicales.

\subsection{Les équations allométriques locales}

Dans la base de données GlobAllometree (www. globallometree.org), qui recense les équations allométriques disponibles pour tous les continents, un total de 1058 équations allométriques de biomasse et de volume ont été répertoriées pour l'Afrique sub-saharienne (Henry et al., 2013). La plupart des équations disponibles sont mono-spécifiques et sont des équations de volume (Henry et al., 2011). Dans les forêts tropicales, qui sont structurellement complexes et riches en espèces, il est illusoire de développer des équations allométriques pour chaque espèce et il apparait indispensable d'avoir recours à des équations plurispécifiques intégrant la densité du bois dans le jeu des prédicteurs (Chave et al., 2005). Actuellement, en Afrique tropicale, peu d'équations de ce type ont été établies selon un protocole adéquat (i.e. $>100$ arbres bien répartis sur la gamme de diamètre, Chave et al., 2004). Leur rareté est encore plus flagrante en ce qui concerne la prise en compte de la biomasse souterraine (Tableau 1).

Certaines équations allométriques de biomasse ont été construites à partir d'un nombre limité d'individus, 12 arbres (Ebuy et al., 2011), 26 arbres (Ryan et al., 2011), 29 arbres (Henry et al., 2009) et 42 arbres (Henry et al., 2010). D'autres intègrent très peu d'arbres de gros diamètres, de 1 à $79 \mathrm{~cm}$ de diamètre (Djomo et al., 2010) et de 1 à $67 \mathrm{~cm}$ de diamètre (Goussanou et al., 2016). Enfin, dans les forêts claires de type Miombo, dans les forêts de type Miombo, en Tanzanie (Mugasha et al., 2013) et intègre l'estimation de la biomasse souterraine.

Toutefois, l'utilisation des équations allométriques locales dans des sites autres que ceux pour lesquels elles ont été développées pourrait entrainer des biais importants. En effet, les équations allométriques développées au Mozambique (Ryan et al., 2011), au Kenya (Kuyah et al., 2012a), en Tanzanie (Mugasha et al., 2013) et en Zambie (Chidumayo, 2013) entrainent respectivement des biais de 24, 29, 50 et $72 \%$ de biomasse aérienne à l'échelle des forêts de type Miombo au Malawi (Kuyah et al., 2014). De même, Picard et al. (2015) estiment des biais respectifs de 29 et $33 \%$ dans les estimations de la biomasse aérienne à l'échelle de la réserve forestière de Yoko en RDC à partir des équations provenant du Cameroun (Fayolle et al., 2013) et du Gabon (Ngomanda et al., 2014).

Cependant, deux études utilisant la méthode indirecte de l'estimation de la biomasse reposent sur l'estimation du volume de la tige (Maliro et al., 2010) et du volume total de l'arbre (Dorisca et al., 2011). Leur utilisation avec des facteurs de conversion (densité du bois et facteur d'expansion de la biomasse) entrainerait une surestimation de la biomasse de $19 \%$ au Gabon (Moundounga Mavouroulou et al., 2014). Picard et al. (2015) montrent également des biais d'environ $48 \%$ dans les estimations de la biomasse aérienne à l'échelle de la réserve forestière de Yoko en RDC à partir des équations allométriques de volume de Dorisca et al. (2011).

\section{ESTIMATION DE LA BIOMASSE AU NIVEAU DU PEUPLEMENT}

\subsection{Variation spatiale de la biomasse}

Il existe une forte variation spatiale de la biomasse au sein des forêts tropicales et notamment entre les 


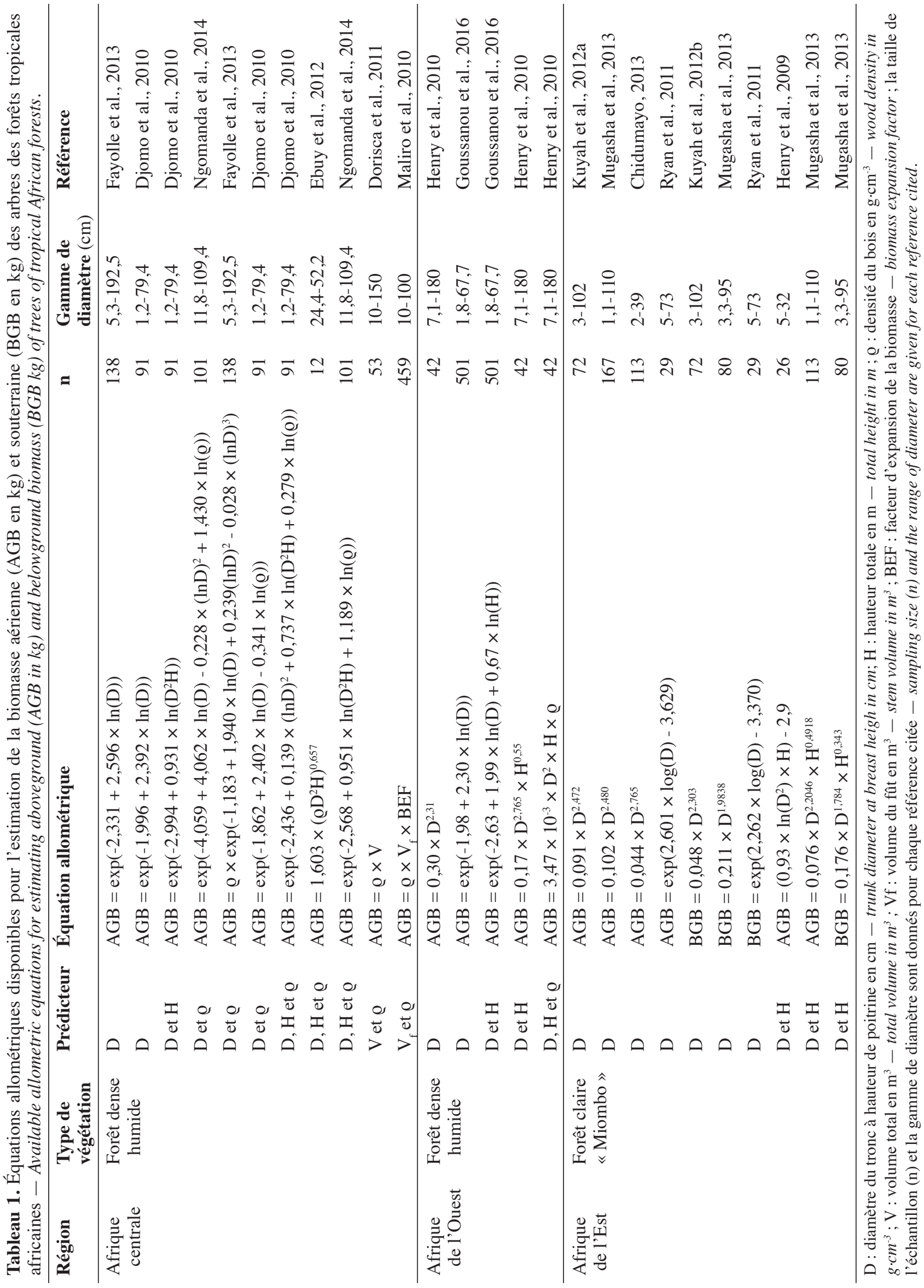


trois bassins forestiers tropicaux avec des valeurs plus élevées en Afrique tropicale et en Asie, respectivement $418 \pm 91$ et $393 \pm 109 \mathrm{Mg} \cdot \mathrm{ha}^{-1}$, que dans les forêts d'Amérique du Sud, $287 \pm 105 \mathrm{Mg} \cdot \mathrm{ha}^{-1}$ (Slik et al., 2013). Ces variations s'expliqueraient par la fréquence plus élevée d'arbres de plus de $70 \mathrm{~cm}$ de diamètre dans les forêts paléotropicales (Afrique et Asie). Au sein du continent africain, il existe aussi d'importantes variations spatiales de biomasse. Lewis et al. (2013) indiquent des estimations de biomasse en Afrique centrale $\left(429 \mathrm{Mg} \cdot \mathrm{ha}^{-1}\right)$ nettement supérieures à celles d'Afrique de l'Ouest (305 Mg.ha' ${ }^{-1}$ ) et de l'Est (274 Mg. $\left.\mathrm{ha}^{-1}\right)$. Ces différences de biomasse s'expliqueraient par la présence élevée d'espèces hyperdominantes en Afrique centrale, qui contribuent à plus de $50 \%$ des stocks de biomasse (Bastin et al., 2015).

À l'échelle locale, plusieurs auteurs ont également montré des variations significatives de la biomasse entre les différents types de forêts tropicales africaines (Tableau 2). Day et al. (2013) indiquent des variations de la biomasse aérienne entre les différents types de forêts denses humides d'Afrique centrale et Kuyah et al. (2014) entre les types de forêts de Miombo d'Afrique de l'Est. Ces variations sont essentiellement expliquées par des différences structurales liées à des perturbations anthropiques et/ou des gradients édaphiques et altitudinaux. Toutefois, la composition floristique et les variables structurales (surface terrière, allométrie hauteur-diamètre, etc.) expliquent une plus grande partie de la variation spatiale de la biomasse dans les forêts tropicales africaines (Marshall et al., 2012 ; Shirima et al., 2015).

D'une part, les variations spatiales de la biomasse s'expliqueraient par des compositions spécifiques différentes des types de forêts (Djomo et al., 2011; Marshall et al., 2012 ; Bastin et al., 2014 ; Ekoungoulou et al., 2015 ; Shirima et al., 2015 ; Wondrade et al., 2015). Les forêts matures où Gilbertiodendron dewevrei (Fabaceae - Caesalpinioideae) forment des peuplements mono-dominants stockent autant voire plus de biomasse aérienne que les forêts mixtes plus jeunes au Cameroun (Djuikouo et al., 2010) et en RDC (Makana et al., 2011; Kearsley et al., 2013). Selon Maniatis et al. (2011), les forêts dominées par des arbres des familles Olacaceae, Caesalpiniaceae et Burseraceae présentent une biomasse aérienne nettement supérieure à celle des forêts dominées par des familles Burseraceae, Myristicaceae et Euphorbiaiceae.

D'autre part, Fayolle et al. (2016) indiquent que les variations spatiales de la biomasse aérienne entre les forêts sempervirentes et semi-décidues au Cameroun s'expliqueraient par les différences de la composition floristique, de la structure forestière (densité des tiges à l'hectare et la surface terrière) et de l'allométrie hauteur-diamètre. Une étude réalisée en RDC dans les forêts semi-décidues de Yangambi et de Yoko montre que l'allométrie hauteur-diamètre est le facteur qui expliquerait le plus de différence dans les variations spatiales de la biomasse aérienne (Doetterl et al., 2015).

L'interaction entre la composition floristique, la structure forestière et les facteurs de l'environnement (sols) expliquerait les différences de biomasse aérienne entre les forêts centrafricaines matures sur sols riches ou sur sols pauvres et celles sur sols présentant des contraintes physiques (Gourlet-Fleury et al., 2011). D'autres facteurs de l'environnement pourraient également influencer la répartition spatiale de la biomasse. Les forêts de type Miombo de moyenne altitude stockent plus de biomasse par rapport à celles de basse et haute altitude (Shirima et al., 2011 ; Marshall et al., 2012). Les précipitations expliquent une grande part des variations spatiales de la biomasse entre les types de forêts de montagne en Tanzanie (Ensslin et al., 2015).

\subsection{Variation temporelle de la biomasse}

Au cours de ces dernières années, plusieurs études ont montré une tendance à l'augmentation de la biomasse aérienne et des stocks de carbone dans les forêts tropicales matures/non perturbées (Phillips et al., 1994), y compris en Afrique (Lewis et al., 2009 ; Gourlet-Fleury et al., 2013). Elles se comporteraient comme des puits de carbone.

Ainsi, Makana et al. (2011) ont montré que la biomasse a augmenté en moyenne de $1,1 \mathrm{Mg} \cdot \mathrm{ha}^{-1} \cdot \mathrm{an}^{-1}$ dans les forêts mono-dominantes à Gilbertiodendron dewevrei, forêt mature et de $3 \mathrm{Mg} \cdot \mathrm{ha}^{-1} \cdot \mathrm{an}^{-1}$ dans les forêts mixtes (forêt jeune) d'Ituri en RDC sur 12 années. Cette différence s'expliquerait par une plus faible vitesse de croissance des arbres dans les forêts mono-dominantes, où le degré de maturité est plus avancé. Toutefois, la biomasse des forêts matures est supérieure à celle des forêts jeunes (Glenday, 2006 ; Kearsley et al., 2013).

Cependant, les activités humaines, dont l'agriculture sur brûlis et l'exploitation forestière, affectent inévitablement la biomasse et les stocks de carbone (Lindsell et al., 2013 ; Willcock et al., 2014). En Afrique centrale, les forêts tropicales couvrent environ 180 millions d'hectares et $26 \%$ de cette superficie est valorisé pour un usage commercial par les sociétés d'exploitation forestière (de Wasseige et al., 2012). L'exploitation forestière sélective, telle qu'elle est pratiquée dans cette sous-région, se traduit par une réduction de 17 à $20 \mathrm{Mg} \cdot \mathrm{ha}^{-1}$ de biomasse initiale (Brown et al., 2005 ; Medjibe et al., 2011). Ces pertes de biomasse étant temporelles, l'optimisation des tracés des pistes de débardage pourrait agir significativement sur la réduction à ces pertes de biomasse liées à l'exploitation forestière (de Madron et al., 2011 ; Neba et al., 2014). Une période 


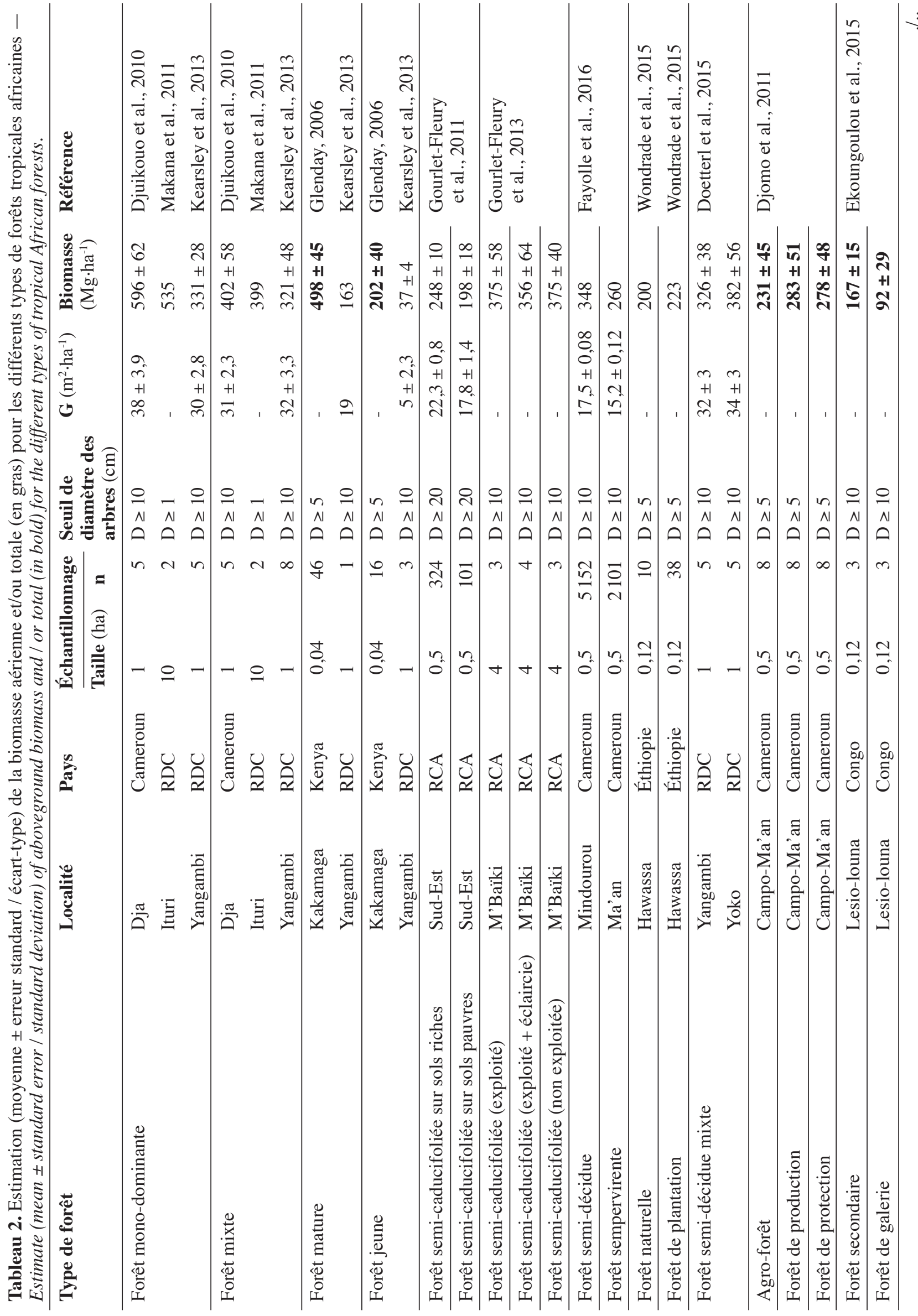


Biomasse et carbone forestier en Afrique tropicale

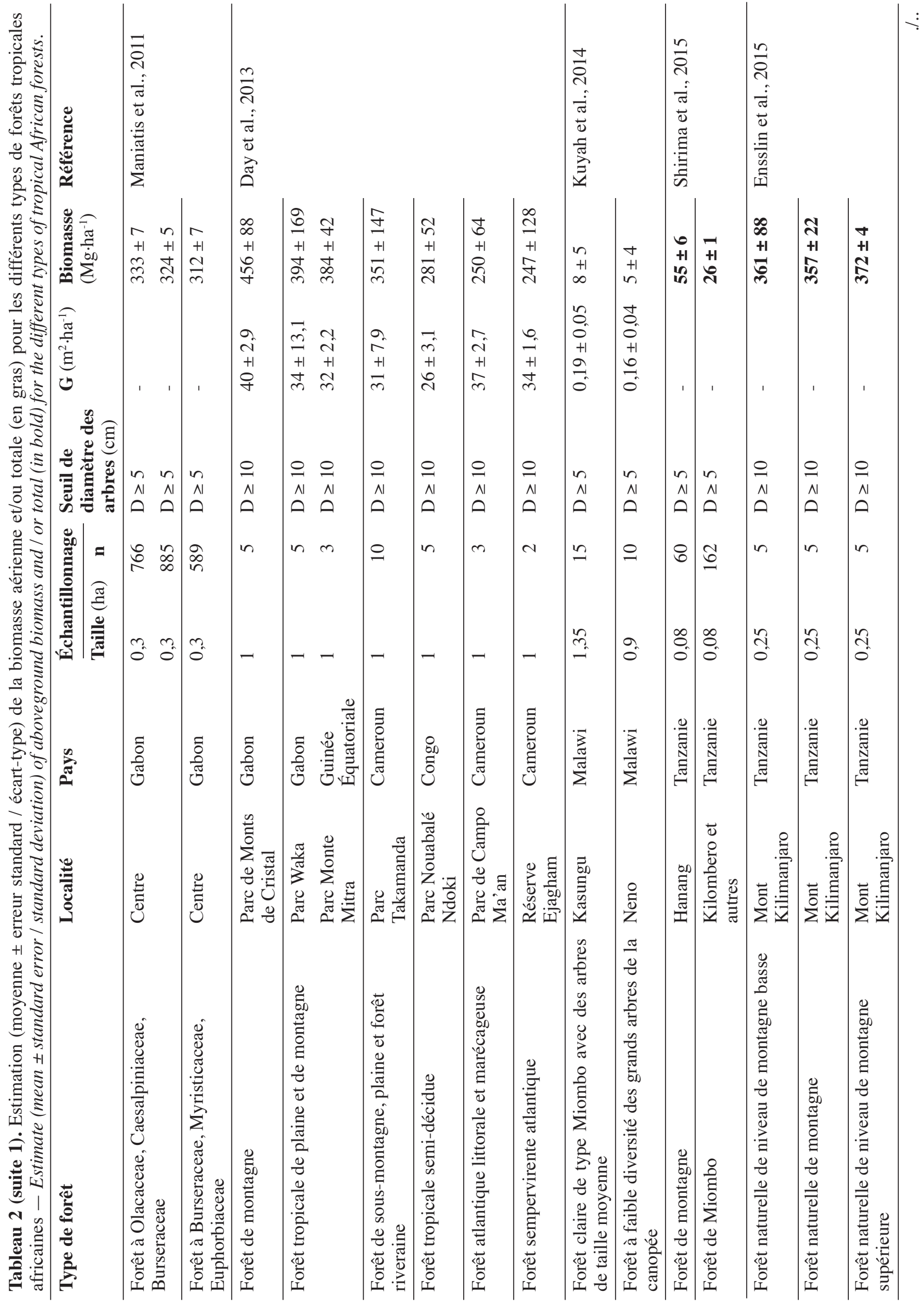


d'environ 30 ans serait cependant nécessaire pour la reconstitution des stocks initiaux de biomasse en Afrique centrale (GourletFleury et al., 2013 ; Ndjondo et al., 2014).

La vitesse de reconstitution de la biomasse forestière après l'exploitation semble être influencée par l'intensité et le type d'exploitation forestière (Gourlet-Fleury et al., 2013; Medjibe et al., 2013; Neba et al., 2014). Toutefois, Lung et al. (2015) n'ont pas trouvé de différence de biomasse entre quatre régions du Kenya ayant subi des techniques d'exploitation différentes. De même, les stocks de carbone dans les forêts exploitées ne sont pas différents de ceux des forêts non exploitées en Tanzanie (Marshall et al., 2012 ; Ensslin et al., 2015). Gatti et al. (2015) indiquent également que la biomasse des forêts exploitées n'est pas différente de celle des forêts primaires dans les forêts tropicales africaines.

\section{CARTOGRAPHIE DE LA BIOMASSE}

\subsection{Différents capteurs}

Compte tenu de l'étendue des forêts tropicales ainsi que de la difficulté d'accès, les méthodes de télédétection sont depuis longtemps considérées comme des outils essentiels pour produire des cartes de biomasse à plus ou moins grande échelle (Gibbs et al., 2007 ; Clark et al., 2012). Actuellement, les principaux outils de télédétection utilisés pour la cartographie de la biomasse en Afrique tropicale sont: les capteurs optiques, le RADAR (Radio Detection And Ranging) et le LiDAR (Light Detection and Ranging) (Tableau 3).

Les données optiques les plus couramment utilisées pour estimer la biomasse sont les images Landsat (Avitabile et al., 2012), SPOT (Satellites Pour l'Observation de la Terre) (Verhegghen et al., 2012) et MODIS (MODerate resolution Imaging Spectroradiometer) (Baccini et al., 2008). Une haute résolution spatiale des capteurs optiques augmente la précision des estimations de la biomasse. Le problème majeur serait la forte présence de nuages sous les tropiques qui limite le nombre déjà faible d'images de haute résolution disponibles (Gibbs et al., 2007 ; Goetz et al., 2009). Les images de très haute résolution spatiale 
provenant des capteurs satellitaires (par exemple: Quickbird, GeoEye) peuvent également être utilisées pour estimer les stocks de carbone forestier avec une faible erreur sur des zones relativement restreintes (Bastin et al., 2014).

À large échelle, l'utilisation du RADAR semble prometteuse pour la cartographie de la biomasse en raison de sa capacité à pénétrer le couvert forestier à une certaine profondeur (Goetz et al.,2009). Plusieurs études récentes en Afrique tropicale ont utilisé le RADAR pour l'estimation de la biomasse (Mitchard et al., 2009 ; Carreiras et al., 2012 ; Mitchard et al., 2012 ; Carreiras et al., 2013; Mermoz et al., 2015). Cependant, le signal RADAR souffre de problèmes de saturation sur des sites forestiers à forte densité de biomasse, comme en forêts tropicales (Goetz et al., 2009), y compris dans les forêts tropicales africaines (Mitchard et al., 2009). Ce problème de saturation du signal RADAR entrainerait des sous-estimations de la biomasse dans les forêts tropicales.

Le LiDAR, qui est un capteur actif comme le RADAR, présente l'avantage de ne pas saturer pour les fortes biomasses mais le cout d'acquisition de ce type de données reste un frein majeur à son utilisation (Lu et al., 2014). Une revue bibliographique de 70 études indique que le LiDAR est nettement plus performant pour estimer la biomasse forestière que les capteurs optiques ou RADAR utilisés seuls (Zolkos et al., 2013). L'utilisation du LiDAR en Afrique tropicale pourrait donc améliorer significativement les estimations de biomasse (Avitabile et al., 2012 ; Mitchard et al., 2012 ; Laurin et al., 2014 ; Chen et al., 2015). Des systèmes LiDAR satellitaires permettraient de diminuer ce cout. Actuellement, le seul système LiDAR satellitaire existant est le GLAS (Geoscience Laser Altimeter System) du satellite ICESat qui a fonctionné en intermittence de 2003 à 2009. Le capteur a néanmoins permis de récolter près de 250 millions de faisceaux LiDAR relatifs à des zones forestières dont 18,2 millions de faisceaux ont été acquis en Afrique tropicale. L'information déduite de ces faisceaux a permis d'effectuer une cartographie de la biomasse des forêts tropicales (Saatchi et al., 2011 ; Baccini et al., 2012).

Tous les types de capteur présentés dans cette section ont leurs avantages et inconvénients. La combinaison des données provenant des différents capteurs permet, par ailleurs, de bénéficier des avantages de chacun et, in fine, de réduire les erreurs liées à l'estimation de la biomasse par rapport aux estimations basées sur un seul type de capteur. Par exemple, l'intégration des capteurs optiques à distance (bandes hyper-spectrales) augmente la précision du modèle $\left(\mathrm{R}^{2}=0,70\right)$ par rapport au modèle basé sur le LiDAR seul $\left(\mathrm{R}^{2}=0,64\right)$ dans l'étude locale de Laurin et al. (2014) sur une forêt 
tropicale du Sud de la Sierra Leone. Par ailleurs, la plupart des dernières cartes de biomasse sur des grandes étendues sont produites à partir de données issues de plusieurs capteurs (Saatchi et al., 2011 ; Baccini et al., 2012 ; Mitchard et al., 2012).

\subsection{Carte de biomasse : les produits actuels et leurs limites}

La qualité de la cartographie de la biomasse dépend fortement des types de capteurs utilisés (Optique, RADAR ou LiDAR), de la résolution spatiale et/ ou temporelle des données de ces capteurs (Zolkos et al., 2013), du plan d'échantillonnage des inventaires forestiers, des estimations de biomasse de terrain utilisées pour calibrer les données de télédétection (Mauya et al., 2015).

À l'échelle locale, une corrélation de 0,95 a été obtenue entre une carte de biomasse établie à partir de données satellitaires RADAR et l'estimation de la biomasse provenant des inventaires forestiers en Guinée-Bissau (Carreiras et al., 2012) et au Mozambique (Carreiras et al., 2013). Selon Avitabile et al. (2012), le modèle développé pour estimer la biomasse à l'échelle nationale en Ouganda à partir d'images Landsat présente une précision élevée $\left(R^{2}=\right.$ $0,81)$. Cette importante précision est liée à la faible densité de biomasse rencontrée dans le pays (moins $25 \mathrm{Mg} \cdot \mathrm{ha}^{-1}$ sur $87 \%$ du territoire). En RDC, Bastin et al. (2014) indiquent une bonne relation $\left(R^{2}=\right.$ $0,85)$ entre la biomasse estimée à partir des données d'inventaires forestiers et la biomasse prédite sur base de la texture forestière analysée à partir d'images de très haute résolution spatiale sur une superficie de $400 \mathrm{~km}^{2}$.

Toutefois, Mermoz et al. (2015) indiquent des sous-estimations de la biomasse provenant de données RADAR $\left(109 \pm 42 \mathrm{t} \cdot \mathrm{ha}^{-1}\right)$ par rapport à la biomasse estimée à partir des inventaires forestiers $(238 \pm 30 \mathrm{t}$. $\mathrm{ha}^{-1}$ ) réalisés dans les forêts centrafricaines. Cette sous-estimation serait liée à l'atténuation du signal de la bande L dans les forêts à haute densité de biomasse. Dans les forêts du Ghana, une erreur de prédiction de $20 \%$ est obtenue sur l'estimation de la biomasse aérienne à partir de données LiDAR aériennes (Chen et al., 2015). Finalement, une incertitude d'environ $25 \%$ a été trouvée sur l'estimation de la biomasse à partir de la fusion des données de RADAR et de LiDAR au niveau du Parc national Lopé au Gabon (Mitchard et al., 2012).

À l'échelle régionale, la première carte de biomasse pour les forêts tropicales africaines a été produite par Baccini et al. (2008) à partir de données MODIS et largement critiquée par la suite. Les valeurs de la biomasse provenant de la carte de Baccini et al. (2008) ne correspondent pas à celles provenant de la carte
d'Avitabile et al. (2012) en Ouganda. Selon Mitchard et al. (2011), les prédictions de la carte de Baccini et al. (2008) ne correspondent pas à celles d'un ensemble de données d'inventaires forestiers indépendants de 1154 parcelles de 16 pays africains. La comparaison de valeurs de biomasse provenant de la compilation de données d'inventaire de terrain avec les produits de télédétection reste faible (Mitchard et al., 2009 ; Verhegghen et al., 2012). La synchronisation temporelle entre la collecte des données de terrain (inventaires forestiers) et l'acquisition des données satellitaires, ainsi que l'agrégation spatiale des données terrain à la résolution des données satellitaires sont des points essentiels pour la calibration des cartes de biomasse (Baccini et al., 2011).

À l'échelle pantropicale, deux cartes ont été produites à l'aide de données LiDAR satellitaires et d'autres produits de télédétection avec une résolution de $1 \mathrm{~km}$ (Saatchi et al., 2011) et $500 \mathrm{~m}$ (Baccini et al., 2012). En Afrique tropicale, ces deux cartes présentent de grandes discordances. La carte de biomasse de Baccini et al. (2012) prédit de fortes biomasses en RDC, tandis que celle de Saatchi et al. (2011) prédit de plus fortes biomasses au Gabon. Ces différences s'expliqueraient par le choix de l'équation allométrique utilisée pour convertir les données d'inventaires forestiers en biomasse aérienne, par le traitement des données LiDAR et par les techniques de modélisation spatiale utilisées (Mitchard et al., 2013 ; Langner et al., 2014).

\section{CONCLUSIONS ET PERSPECTIVES}

De cette synthèse bibliographique, il ressort que les stocks de biomasse et de carbone contenus dans les forêts tropicales africaines sont encore incertains. Si les estimations de la biomasse aérienne à l'échelle de l'arbre et du peuplement sont assez bien documentées, cette synthèse démontre que la littérature relative aux estimations de la biomasse souterraine est très pauvre, surtout pour les forêts denses humides d'Afrique centrale. Or, la biomasse souterraine pourrait représenter jusqu'à $20 \%$ de la biomasse totale (Mokany et al., 2006).

Les méthodes d'estimation de la biomasse, qu'elles soient basées sur des données de terrain (inventaires forestiers) ou de télédétection (imagerie optique satellite ou aéroportée, LiDAR, RADAR), dépendent toutes de données d'inventaires combinées aux équations allométriques pour la calibration (Gibbs et al., 2007). Le choix de l'équation allométrique est une étape cruciale dans l'estimation de la biomasse et des stocks de carbone. Néanmoins, peu d'informations sont disponibles sur les variations de l'allométrie de biomasse en Afrique tropicale. Il est alors nécessaire 
d'identifier et/ou de développer des sites de référence pour lesquels des données fiables sur l'allométrie des arbres et des inventaires forestiers à large échelle spatiale sont disponibles. Les inventaires forestiers nationaux (IFN), fournissant une estimation de la biomasse à l'échelle nationale, pourraient donc constituer un complément aux méthodes par télédétection.

Pour améliorer les estimations de la biomasse et du carbone forestier en Afrique tropicale, les thématiques suivantes devraient être prioritairement abordées :

- l'amélioration des équations allométriques locales en ayant recours à une méthodologie standardisée et en incorporant dans les modèles de nouveaux prédicteurs caractérisant l'arbre (c'est le cas, par exemple, de la composante $2 \mathrm{~b}$ du projet PreREDD+ en Afrique centrale) ;

- la mise au point de techniques non destructives utilisant le LiDAR terrestre (Terrestrial Laser Scanning - TLS) pour permettre de reconstituer l'enveloppe d'un arbre en trois dimensions afin de réduire les incertitudes liées aux estimations de la biomasse utilisant le volume des arbres et les équations allométriques ;

- la compréhension des relations allométriques entre les dimensions des arbres et l'allocation de la biomasse au sein d'un arbre, et des variations de l'architecture des arbres entre les types de forêts et entre les espèces ;

- la quantification des erreurs liées à l'estimation de la biomasse à l'échelle de la parcelle et du paysage.

Aborder ces aspects est nécessaire pour calibrer les données de la télédétection et produire les cartes de biomasse fiables dans le cadre du système MNV, fondamental pour la mise en œuvre effective du programme REDD+ en Afrique tropicale.

\section{Remerciements}

Les auteurs remercient la République du Congo à travers l'Office de Gestion des Étudiants et Stagiaires (OGES), la Fondation Internationale pour la Science (IFS), l'Université Marien Ngouabi, l'Université de Liège, l'asbl Nature+, pour leur soutien technique, scientifique et/ou financier. La rédaction de ce manuscrit a été stimulée lors des discussions des projets PreREDD+ et DynAfFor. Que Charles Bracke, Jean-Yves Devleeschouwer, Félicien Tosso et Richard Fousseni Fétéké soient remerciés pour leurs conseils et contributions.

\section{Bibliographie}

Angelsen A., Brockhaus M., Sunderlin W.D. \& Verchot L.V., 2013. Analyse de la REDD+: les enjeux et les choix. Bogor, Indonésie : CIFOR.
Avitabile V. et al., 2011. Mapping biomass with remote sensing: a comparison of methods for the case study of Uganda. Carbon Balance Manage., 6(7), 1-14.

Avitabile V. et al., 2012. Capabilities and limitations of Landsat and land cover data for aboveground woody biomass estimation of Uganda. Remote Sens. Environ., 117, 366-380.

Baccini A. et al., 2008. A first map of tropical Africa's above-ground biomass derived from satellite imagery. Environ. Res. Lett., 3(4), 045011.

Baccini A. et al., 2011. Reply to comment on "A first map of tropical Africa's above-ground biomass derived from satellite imagery". Environ. Res. Lett., 6(4), ID 049002.

Baccini A. et al., 2012. Estimated carbon dioxide emissions from tropical deforestation improved by carbon-density maps. Nat. Clim. Change, 2(3), 182-185.

Bastin J.-F. et al., 2014. Aboveground biomass mapping of African forest mosaics using canopy texture analysis: towards a regional approach. Ecol. Appl., 24(8), 19842001.

Bastin J.-F. et al., 2015. Seeing Central African forests through their largest trees. Sci. Rep., 5, ID 13156.

Brown S., Gillespie A.J.R. \& Lugo A.E., 1989. Biomass estimation methods for tropical forests with applications to forest inventory data. For. Sci., 35(4), 881-902.

Brown S. et al., 2005. Impact of selective logging on the carbon stock of tropical forests: Republic of Congo as a case study. Arlington, VI, USA: Winrock International.

Carreiras J.M.B., Vasconcelos M.J. \& Lucas R.M., 2012. Understanding the relationship between aboveground biomass and ALOS PALSAR data in the forests of Guinea-Bissau (West Africa). Remote Sens. Environ., 121, 426-442.

Carreiras J.M.B., Melo J.B. \& Vasconcelos M.J., 2013. Estimating the above-ground biomass in Miombo savanna woodlands (Mozambique, East Africa) using L-band synthetic aperture radar data. Remote Sens., 5(4), 1524-1548.

Chave J. et al., 2004. Error propagation and scaling for tropical forest biomass estimates. Philos. Trans. R. Soc. London, Ser. B, 359(1443), 409-420.

Chave J. et al., 2005. Tree allometry and improved estimation of carbon stocks and balance in tropical forests. Oecologia, 145(1), 87-99.

Chave J. et al., 2014. Improved allometric models to estimate the aboveground biomass of tropical trees. Global Change Biol., 20, 3177-3190.

Chen Q., Vaglio Laurin G. \& Valentini R., 2015. Uncertainty of remotely sensed aboveground biomass over an African tropical forest: Propagating errors from trees to plots to pixels. Remote Sens. Environ., 160, 134-143.

Chidumayo E.N., 2013. Forest degradation and recovery in a miombo woodland landscape in Zambia: 22 years of observations on permanent sample plots. For. Ecol. Manage., 291, 154-161. 
Clark D.B. \& Kellner J.R., 2012. Tropical forest biomass estimation and the fallacy of misplaced concreteness. J. Veg. Sci., 23, 1191-1196.

Day M. et al., 2013. Relationships between tree species diversity and above-ground biomass in Central African rainforests: implications for REDD. Environ. Conserv., 41, 64-72.

de Madron L.D. et al., 2011. Estimation de l'impact de différents modes d'exploitation forestière sur les stocks de carbone en Afrique centrale. Bois For. Trop., 308(2), 75-86.

de Wasseige C. et al., 2012. Les forêts $d u$ bassin $d u$ Congo. État des Forêts 2010. Luxembourg: Office des publications de l'Union européenne.

Djomo A.N. et al., 2010. Allometric equations for biomass estimations in Cameroon and pan moist tropical equations including biomass data from Africa. For. Ecol. Manage., 260, 1873-1885.

Djomo A.N., Knohl A. \& Gravenhorst G., 2011. Estimations of total ecosystem carbon pools distribution and carbon biomass current annual increment of a moist tropical forest. For. Ecol. Manage., 261(8), 1448-1459.

Djuikouo K. et al., 2010. Diversity and aboveground biomass in three tropical forest types in the Dja Biosphere Reserve, Cameroon. Afr. J. Ecol., 48, 1053-1063.

Doetterl S. et al., 2015. Aboveground vs. belowground carbon stocks in African tropical lowland rainforest: Drivers and implications. PLoS ONE, 10(11), e0143209.

Dorisca S. et al., 2011. Établissement d'équations entre le diamètre et le volume total de bois des arbres, adaptées au Cameroun. Bois For. Trop., 308(2), 87-95.

Ebuy J. et al., 2011. Allometric equation for predicting aboveground biomass of three tree species. J. Trop. For. Sci., 23(2), 125-132.

Ekoungoulou R. et al., 2015. Evaluating the carbon stock in above-and below-ground biomass in a moist central African forest. Appl. Ecol. Environ. Sci., 3(2), 51-59.

Ensslin A. et al., 2015. Effects of elevation and land use on the biomass of trees, shrubs and herbs at Mount Kilimanjaro. Ecosphere, 6(3), 45.

Fayolle A. et al., 2013. Tree allometry in Central Africa: Testing the validity of pantropical multi-species allometric equations for estimating biomass and carbon stocks. For. Ecol. Manage., 305, 29-37.

Fayolle A. et al., 2016. Taller trees, denser stands and greater biomass in semi-deciduous than in evergreen lowland central African forests. For. Ecol. Manage., 374, 4250.

Feldpausch T.R. et al., 2012. Tree height integrated into pantropical forest biomass estimates. Biogeosciences, 9(8), 3381-3403.

Gatti R.C. et al., 2015. The impact of selective logging and clearcutting on forest structure, tree diversity and aboveground biomass of African tropical forests. Ecol. Res., 30, 119-132.
Gibbs H.K. et al., 2007. Monitoring and estimating tropical forest carbon stocks: making REDD a reality. Environ. Res. Lett., 2(4), 045023.

GIEC, 2003. Good practice guidance for land use, land-use change and forestry. GIEC.

Glenday J., 2006. Carbon storage and emissions offset potential in an East African tropical rainforest. For. Ecol. Manage., 235(1-3), 72-83.

Goetz S.J. et al., 2009. Mapping and monitoring carbon stocks with satellite observations: a comparaison of methods. Carbon Balance Manage., 4, 2.

Goodman R.C., Phillips O.L. \& Baker T.R., 2014. The importance of crown dimensions to improve tropical tree biomass estimates. Ecol. Appl., 24(4), 680-698.

Gourlet-Fleury S. et al., 2011. Environmental filtering of dense-wooded species controls above-ground biomass stored in African moist forests. J. Ecol., 99, 981-990.

Gourlet-Fleury S. et al., 2013. Tropical forest recovery from logging: a 24 year sylvicultural experiment from Central Africa. Philos. Trans. R. Soc.London, Ser. B, 368(1625), 20120302.

Goussanou C.A. et al., 2016. Specific and generic stem biomass and volume models of tree species in a West African tropical semi-deciduous forest. Silva Fennica, $\mathbf{5 0}(2), 1474$.

Henry M. et al., 2009. Biodiversity, carbon stocks and sequestration potential in aboveground biomass in smallholder farming systems of western Kenya. Agric. Ecosyst. Environ., 129, 238-252.

Henry M. et al., 2010. Wood density, phytomass variations within and among trees, and allometric equations in a tropical rainforest of Africa. For. Ecol. Manage., 260, 1375-1388.

Henry M. et al., 2011. Estimating tree biomass of SubSaharan African forests: A review of available allometric equations. Silva Fennica, 45, 477-569.

Henry M. et al., 2013. GlobAllomeTree: international platform for tree allometric equations to support volume, biomass and carbon assessment. iForest Biogeosci. For., 6(5), 326-330.

Kearsley E. et al., 2013. Conventional tree height-diameter relationships significantly overestimate aboveground carbon stocks in the Central Congo Basin. Nat. Commun., 4, 2269.

King D.A., 1996. Allometry and life history of tropical trees. J. Trop. Ecol., 12(1), 25-44.

Kuyah S. et al., 2012a. Allometric equations for estimating biomass in agricultural landscapes: I. Aboveground biomass. Agric. Ecosyst. Environ., 158, 216-224.

Kuyah S. et al., 2012b. Allometric equations for estimating biomass in agricultural landscapes: II. Belowground biomass. Agric. Ecosyst. Environ, 158, 225-234.

Kuyah S. et al., 2014. Estimating aboveground tree biomass in three different miombo woodlands and associated land use systems in Malawi. Biomass Bioenergy, 66, 214-222. 
Langner A., Achard F. \& Grassi G., 2014. Can recent pantropical biomass maps be used to derive alternative Tier 1 values for reporting REDD+ activities under UNFCCC? Environ. Res. Lett., 9(12), 124008.

Laurin G.V. et al., 2014. Above ground biomass estimation in an African tropical forest with lidar and hyperspectral data. ISPRS J. Photogramm. Remote Sens., 89, 49-58.

Lewis S.L. et al., 2009. Increasing carbon storage in intact African tropical forests. Nature, 457, 1003-1006.

Lewis S.L. et al., 2013. Aboveground biomass and structure of 260 African tropical forests. Philos. Trans. R. Soc. London, Ser. B, 368(1625), 20120295.

Lindsell J.A. \& Klop E., 2013. Spatial and temporal variation of carbon stocks in a lowland tropical forest in West Africa. For. Ecol. Manage., 289, 10-17.

Lu D. et al., 2014. A survey of remote sensing-based aboveground biomass estimation methods in forest ecosystems. Int. J. Digital Earth, 9(1), 1-43, doi: 10.1080/17538947.2014.990526.

Lung M.\& Espira A., 2015. The influence of stand variables and human use on biomass and carbon stocks of a transitional African forest: Implications for forest carbon projects. For. Ecol. Manage., 351, 36-46.

Makana J.-R. et al., 2011. Demography and biomass change in monodominant and mixed old-growth forest of the Congo. J. Trop. Ecol., 27(05), 447-461.

Maliro T.K., Lokombe Dimandja J.-P. \& Picard N., 2010. Volume equations and biomass estimates for three species in tropical moist forest in the Orientale province, Democratic Republic of Congo. South. For., 72(3/4), 141-146.

Maniatis D. et al., 2011. Evaluating the potential of commercial forest inventory data to report on forest carbon stock and forest carbon stock changes for REDD+ under the UNFCCC. Int. J. For. Res., ID 134526.

Marshall A.R. et al., 2012. Measuring and modeling aboveground carbon and tree allometry along a tropical elevation gradient. Biol. Conserv., 154, 20-33.

Mauya E.W. et al., 2015. Effects of field plot size on prediction accuracy of aboveground biomass in airborne laser scanning-assisted inventories in tropical rain forests of Tanzania. Carbon Balance Manage., 10(1), 10.

Medjibe V.P. et al., 2011. Impacts of selective logging on above-ground forest biomass in the Monts de Cristal in Gabon. For. Ecol. Manage., 262, 1799-1806.

Medjibe V.P., Putz F.E. \& Romero C., 2013. Certified and uncertified logging concessions compared in Gabon: changes in stand structure, tree species, and biomass. Environ. Manage., 51(3), 524-540.

Mermoz S. et al., 2015. Decrease of L-band SAR backscatter with biomass of dense forests. Remote Sens. Environ., 159, 307-317.

Mitchard E.T.A. et al., 2009. Using satellite radar backscatter to predict above-ground woody biomass: A consistent relationship across four different African landscapes. Geophys. Res. Lett., 36(23), L23401.
Mitchard E.T.A. et al., 2011. Comment on "A first map of tropical Africa's above-ground biomass derived from satellite imagery". Environ. Res. Lett., 6(4), 049001.

Mitchard E.T.A. et al., 2012. Mapping tropical forest biomass with radar and space borne LiDAR in Lopé National Park, Gabon: overcoming problems of high biomass and persistent cloud. Biogeosciences, 9(1), 179191.

Mitchard E.T.A. et al., 2013. Uncertainty in the spatial distribution of tropical forest biomass: a comparison of pan-tropical maps. Carbon Balance Manage., 8(1), 110.

Mokany K., Raison R.J. \& Prokushkin A.S, 2006. Critical analysis of root: shoot ratios in terrestrial biomes. Global Change Biol., 12(1), 84-96.

Molto Q., Rossi V. \& Blanc L., 2013. Error propagation in biomass estimation in tropical forests. Methods Ecol. Evol., 4(2), 175-183.

Moundounga Mavouroulou Q. et al., 2014. How to improve allometric equations to estimate forest biomass stocks? Some hints from a central African forest. Can. J. For. Res., 44, 685-691.

Mugasha W.A. et al., 2013. Allometric models for prediction of above- and belowground biomass of trees in the miombo woodlands of Tanzania. For. Ecol. Manage., 310, $87-101$.

Ndjondo M. et al., 2014. Opportunity costs of carbon sequestration in a forest concession in central Africa. Carbon Balance Manage., 9(1), 1-13.

Neba S.G., Kanninen M., Atyi R.E.A. \& Sonwa D.J., 2014. Assessment and prediction of above-ground biomass in selectively logged forest concessions using field measurements and remote sensing data: Case study in South East Cameroon. For. Ecol. Manage., 329, 177185.

Ngomanda A. et al., 2014. Site-specific versus pantropical allometric equations: Which option to estimate the biomass of a moist central African forest? For. Ecol. Manage., 312, 1-9.

Pan Y. et al., 2011. A large and persistent carbon sink in the world's forests. Science, 333, 988-993.

Phillips O.L. \& Gentry A.H., 1994. Increasing turnover through time in tropical forests. Science, 263, 954958.

Picard N., Saint-André L. \& Henry M., 2012. Manuel de construction d'équations allométriques pour l'estimation du volume et la biomasse des arbres : de la mesure de terrain à la prédiction. Rome: FAO ; Montpellier, France : CIRAD.

Picard N., Boyemba Bosela F. \& Rossi V., 2015. Reducing the error in biomass estimates strongly depends on model selection. Ann. For. Sci., 72(6), 811-823.

Ploton P.et al., 2016. Closing a gap in tropical forest biomass estimation: taking crown mass variation into account in pantropical allometries. Biogeosciences, 13(5), 15711585 . 
Ryan C.M., Williams M. \& Grace J., 2011. Above- and belowground carbon stocks in a miombo wooodland landscape of Mozambique. Biotropica, 43(4), 423-432.

Saatchi S.S. et al., 2011. Benchmark map of forest carbon stocks in tropical regions across three continents. Proc. Natl. Acad. Sci. U.S.A., 108(24), 9899-9904.

Shirima D.D. et al., 2011. Carbon storage, structure and composition of miombo woodlands in Tanzania's Eastern Arc Mountains. Afr. J. Ecol., 49(3), 332-342.

Shirima D.D. et al., 2015. Relationships between tree species richness, evenness and aboveground carbon storage in montane forests and miombo woodlands of Tanzania. Basic Appl. Ecol., 16(3), 239-249.

Slik J.W.F. et al., 2013. Large trees drive forest aboveground biomass variation in moist lowland forests across the tropics. Global Ecol. Biogeogr., 22, 1261-1271.

Stephenson N.L. et al., 2014. Rate of tree carbon accumulation increases continuously with tree size. Nature, 507, 90-93.

van Breugel M. et al., 2011. Estimating carbon stock in secondary forests: Decisions and uncertainties associated with allometric biomass models. For. Ecol. Manage., 262(8), 1648-1657. van der Werf G.R. et al., 2009. $\mathrm{CO}_{2}$ emissions from forest loss. Nat. Geosci., 2(11), 737-738.

Verhegghen A. et al., 2012. Mapping Congo Basin vegetation types from $300 \mathrm{~m}$ and $1 \mathrm{~km}$ multi-sensor time series for carbon stocks and forest areas estimation. Biogeosciences, 9(12), 5061-5079.

Willcock S. et al., 2014. Quantifying and understanding carbon storage and sequestration within the eastern Arc Mountains of Tanzania, a tropical biodiversity hotspot. Carbon Balance Manage., 9, 2.

Wondrade N., Dick O.B. \& Tveite H., 2015. Estimating aboveground biomass and carbon stock in the Lake Hawassa Watershed, Ethiopia by integrating remote sensing and allometric equations. For. Res., 4(151), 2.

Zolkos S.G., Goetz S.J. \& Dubayah R., 2013. A metaanalysis of terrestrial aboveground biomass estimation using lidar remote sensing. Remote Sens. Environ., 128, 289-298.

(93 réf.) 